\title{
Controllability of Multiagent Systems with a Directed Tree
}

\author{
Li Wang ${ }^{1}$ and Xiao Han ${ }^{2}$ \\ ${ }^{1}$ Beijing Key Lab of Urban Intelligent Traffic Control Technology, North China University of Technology, Beijing 100144, China \\ ${ }^{2}$ College of Science, North China University of Technology, Beijing 100144, China \\ Correspondence should be addressed to Xiao Han; tgncuthanxiao@163.com
}

Received 12 June 2015; Accepted 16 July 2015

Academic Editor: Michael Z. Q. Chen

Copyright (C) 2015 L. Wang and X. Han. This is an open access article distributed under the Creative Commons Attribution License, which permits unrestricted use, distribution, and reproduction in any medium, provided the original work is properly cited.

\begin{abstract}
This paper addresses the controllability problem of multiagent systems with a directed tree based on the classic agreement protocol, in which the information communication topologies being a directed tree and containing a directed tree are both investigated. Different from the literatures, a new method, the star transform, is proposed to study the controllability of multiagent systems with directed topology. Some sufficient and necessary conditions are given for the controllability of such multiagent system. Numerical examples and simulations are proposed to illustrate the theoretical results.
\end{abstract}

\section{Introduction}

Distributed coordination of multiple dynamic agents systems has become a hot topic of major interest [1-6] in recent years. Studies in this direction have been greatly inspired by the cooperative behavior in nature, such as bird flocks, fish schools, ant swarms, and bacteria colonies [7], as well as being driven by the broad applications in engineering fields [8-16], such as cooperative control of unmanned air vehicles (UAVs), schooling of underwater vehicles, formation control of multirobots, attitude alignment of satellite clusters, and congestion control of communication networks [17].

The controllability issue of multiagent systems is a key problem for coordinated control of multiagent systems and shows new features and difficulties. In general, the controllability of a multiagent network refers to transferring the follower agents of such system from any arbitrary initial states to any final state by controlling dynamics of leader agents under exchanged information between each other, in which the interplay between network topologies and agent dynamics plays an important role related to the controllability. The controllability problems were investigated for singleintegrator kinematics $[17,18]$, double-integrator dynamics [19], and high-order-integrator dynamics [20], respectively.

In 2004, Tanner [17] first studied a simple interconnected system model with a single leader that consists of multiple mobile agents with one-integrator dynamics, interconnected through nearest-neighbor rules. A necessary and sufficient condition was obtained for such system with fixed topology to be controllable by regulating the behaviors of the leader, which is assumed to be able to affect its neighbors but not be affected by other group members. In [18, 21], Liu et al. developed the controllability of the discrete system with switching topology and a single leader. The controllability of multiagent systems with multiple leaders based on fixed topology and switching topology was investigated in [22] and [23], respectively. Furthermore, the controllability of multiagent systems with double-integrator dynamics [19] and high-orderintegrator dynamics [20] is studied.

Notice that the results of [24-31] were studied based on an undirected nearest-neighbor topology. However, for the case of networks with directed topologies, as often encountered in practice, it is very hard to solve the controllability problems due to the complexity of the topology and make the controllability of dynamic networks an nontrivial new problem. To date, very few results available in the literature [32] for the controllability of multiagent systems with directed topologies were found. In the context of multiagent networks, we have focused on studying the controllability of multiagent systems with directed topologies in this paper. Some sufficient and necessary conditions for the controllability of such multiagent system with directed topologies are obtained. Compared to the existing works on the related problems [32], the contributions of this paper are summarized as follows: (1) the 
topology is directed; (2) a novel method, the star transform, is introduced; and (3) the geometric criteria for controllability of such multiagent system are given.

The rest of the paper is organized as follows. Section 2 states the problem formulation and some definitions. Section 3 gives the main results on the controllability. Section 4 presents numerical examples and simulation results. Section 5 summarizes the paper.

\section{Preliminaries and Problem Formulation}

2.1. Preliminaries. In this section, we briefly recall some basic notations and concepts in graph theory [33] which will be used in this paper.

A weighted directed graph $\mathscr{G}=(\mathscr{V}, \mathscr{E}, \mathscr{A})$ consists of a vertex set $\mathscr{V}=\left\{v_{1}, v_{2}, \ldots, v_{N}\right\}$ and an edge set $\mathscr{E}=\left\{\left(v_{i}, v_{j}\right)\right.$ : $\left.v_{i}, v_{j} \in \mathscr{V}\right\}$, where an edge is an ordered pair of distinct vertices of $\mathscr{V}$, and the nonsymmetric weighted adjacency matrix $\mathscr{A}=\left[a_{i j}\right]$, with $a_{i j}>0$ if and only if $e_{i j} \in \mathscr{E}$ and $a_{i j}=0$ if not. If all the elements of $\mathscr{V}$ are unordered pairs, then the graph is called an undirected graph. If $v_{i}, v_{j} \in \mathscr{V}$, and $\left(v_{i}, v_{j}\right) \in \mathscr{E}$, then we say that $v_{i}$ and $v_{j}$ are adjacent or $v_{j}$ is a neighbor of $v_{i}$. The neighborhood set of node $v_{i}$ is denoted by $\mathcal{N}_{i}=\left\{v_{j} \in \mathscr{V}:\left(v_{i}, v_{j}\right) \in \mathscr{E}\right\}$. The number of neighbors of each vertex is its degree. A graph is called complete if every pair of vertices is adjacent. A path of length $r$ from $v_{i}$ to $v_{j}$ in a graph is a sequence of $r+1$ distinct vertices starting with $v_{i}$ and ending with $v_{j}$ such that consecutive vertices are adjacent. If there is a path between any two vertices of $\mathscr{G}$, then $\mathscr{G}$ is connected. A directed tree is a directed graph, where the node without any parent is called root, and the root can be connected to any other nodes through paths. Other nodes have exactly one parent (e.g., Figure 1). A spanning tree of a digraph is a directed tree formed by graph edges that connect all the nodes of the graph. A binary tree of a digraph is a directed tree with at most two children for every node, and each child is designated as its left child or right child. $A k$ ary tree is a directed tree with at most $k$ children for every node. If every node has $k$ children or no children, the $k$ ary tree is called a full $k$-ary tree. The degree matrix $\Delta(\mathscr{G})$ of $\mathscr{G}$ is a diagonal matrix with rows and columns indexed by $\mathscr{V}$, in which the $\left(v_{i}, v_{i}\right)$-entry is the degree of vertex $v_{i}$. The symmetric matrix defined as

$$
L(\mathscr{G})=\Delta(\mathscr{G})-A(\mathscr{G})
$$

is the Laplacian of $\mathscr{G}$. The Laplacian is always symmetric and positive semidefinite, and the algebraic multiplicity of its zero eigenvalue is equal to the number of connected components in the graph. For a weighted directed graph $\mathscr{G}$ with $n$ nodes, the out-degree and in-degree of node $v_{i}$ in a weighted directed graph $\mathscr{G}$ with $n$ nodes are, respectively, defined as

$$
\begin{gathered}
\operatorname{deg}_{\text {out }}\left(v_{i}\right)=\sum_{j=1}^{n} a_{j i}, \\
\operatorname{deg}_{\text {in }}\left(v_{i}\right)=\sum_{j=1}^{n} a_{i j} .
\end{gathered}
$$

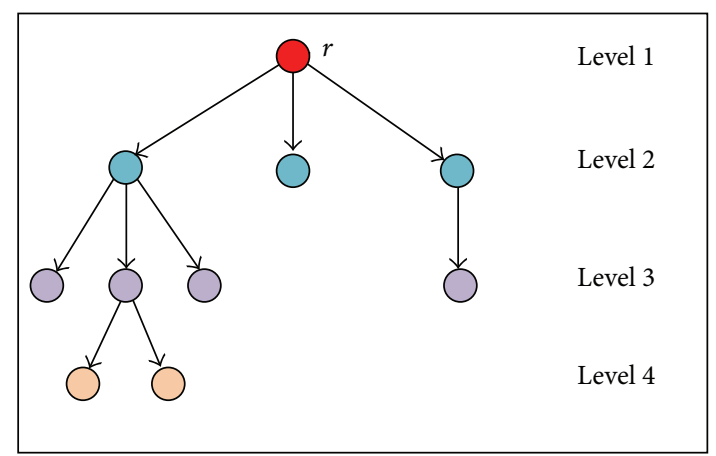

Figure 1: A directed tree.

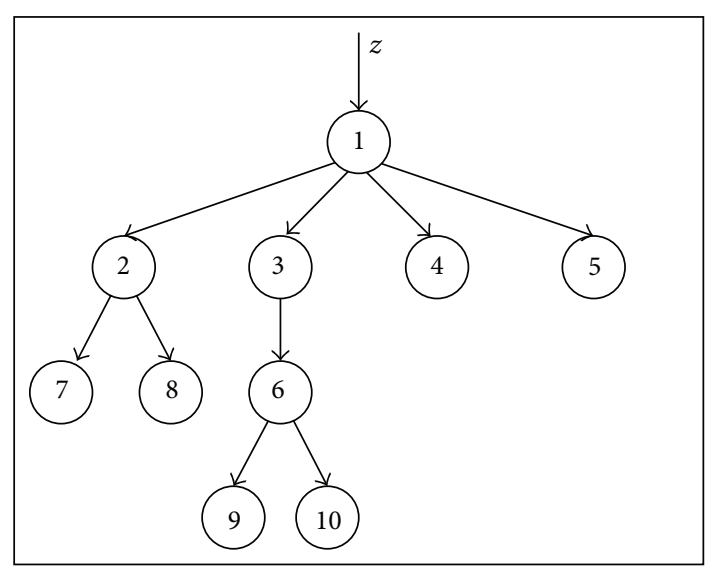

FIgure 2: The topology $\mathscr{G}$.

2.2. Problem Formulation. Consider a multiagent system with a directed topology which is a directed tree, composed of $n$ agents. Choose the first agent (labeled 1) as the root and the remainder $n-1$ agents (labeled from 2 to $n$ ) as children or followers, and each agent moves according to the following dynamics:

$$
\dot{x}_{i}(t)=\sum_{j \in \mathcal{N}_{i}} a_{i j}\left(x_{j}(t)-x_{i}(t)\right)+z_{i}, \quad i=1, \ldots, n,
$$

where $x_{i} \in \mathbb{R}^{1}$ is the state of agent $i$ and $i \in \underline{n}, \underline{n}$ is the index set $(1,2, \ldots, n) ; \mathcal{N}_{i}$ is the neighbor set of agent $i$; $A=\left[a_{i j}\right] \in \mathbb{R}^{m \times m}$ is a matrix describing the interaction or coupling weight between agents, with $a_{i j} \geq 0$ and $a_{i i}=0$. Since the topology is a directed tree, the root agent plays a special role and is responsible for receiving external input or signal and conveying information to the children agents, and hence in model (3), the external input or signal only acts on the root agent (e.g., Figure 2). So, $z_{1} \neq 0, z_{i}=0$, and $a_{1 i}=0$ for $\forall i=2, \ldots, n$.

Let $x=\left(x_{1}, x_{2}, \ldots, x_{n}\right)^{T}$ be the state vector of all the agents; then system (3) follows that

$$
\dot{x}=A x+b z_{1}
$$


where

$$
\begin{aligned}
& A=\left[\begin{array}{cc}
0 & \mathbf{0}_{1 \times(n-1)} \\
A_{1(n-1) \times 1} & A_{e(n-1) \times(n-1)}
\end{array}\right] \in R^{n \times n}, \\
& b=\left[\begin{array}{c}
1 \\
\mathbf{0}_{(n-1) \times 1}
\end{array}\right] \in R^{n \times 1},
\end{aligned}
$$

with

$$
\begin{aligned}
& A_{1}=\left[a_{21}, a_{31}, \ldots, a_{n 1}\right]^{T} \in R^{(n-1) \times 1}, \\
& A_{e}=\left[\begin{array}{ccc}
a_{22} & \cdots & a_{2 n} \\
\vdots & \ddots & \vdots \\
a_{n 2} & \cdots & a_{n n}
\end{array}\right] \in R^{(n-1) \times(n-1)} .
\end{aligned}
$$

Define a star transform matrix $S$ as

$$
S=\left[\begin{array}{ccccc}
1 & 0 & 0 & \cdots & 0 \\
1 & -1 & 0 & \cdots & 0 \\
\vdots & \vdots & \vdots & \ddots & \vdots \\
1 & 0 & 0 & \cdots & -1
\end{array}\right]
$$

It can be easily seen that $S=S^{-1}$, and for the convenience of matrix operations, we partition $S$ as

$$
S=\left[\begin{array}{cc}
1 & \mathbf{0}_{1 \times(n-1)} \\
\mathbf{l}_{(n-1) \times 1} & S_{e(n-1) \times(n-1)}
\end{array}\right] .
$$

Obviously, $S_{e} \in R^{(n-1) \times(n-1)}$ is a negative identity matrix. Denote $y=\left[y_{1}, y_{e}^{T}\right]^{T}$, where $y_{e}=\left[y_{2}, y_{3}, \ldots, y_{n}\right]^{T}$. Let

$$
y=S x
$$

and then we can get $y_{1}=x_{1}$ and $y_{e}=\left[x_{1}-x_{2}, x_{1}-x_{3}, \ldots, x_{1}-\right.$ $\left.x_{n}\right]^{T}$. Therefore, we can have an equivalent system of system (4) as follows:

$$
\dot{y}=S A S^{-1} y+S b z_{1} \text {. }
$$

According to block matrix multiplication, we can have

$$
\begin{aligned}
& \dot{y}_{1}=\left[\begin{array}{ll}
1 & \mathbf{0}_{1 \times(n-1)}
\end{array}\right]\left[\begin{array}{cc}
0 & \mathbf{0}_{1 \times(n-1)} \\
A_{1(n-1) \times 1} & A_{e(n-1) \times(n-1)}
\end{array}\right]\left[\begin{array}{cc}
1 & \mathbf{0}_{1 \times(n-1)} \\
\mathbf{l}_{(n-1) \times 1} & S_{e(n-1) \times(n-1)}
\end{array}\right]\left[\begin{array}{l}
y_{1} \\
y_{e}
\end{array}\right]+\left[\begin{array}{ll}
1 & \mathbf{0}_{1 \times(n-1)}
\end{array}\right]\left[\begin{array}{c}
1 \\
\mathbf{0}_{(n-1) \times 1}
\end{array}\right] z_{1} \\
& =\left[\begin{array}{ll}
0 & \mathbf{0}_{1 \times(n-1)}
\end{array}\right]\left[\begin{array}{cc}
1 & \mathbf{0}_{1 \times(n-1)} \\
\mathbf{1}_{(n-1) \times 1} & S_{e(n-1) \times(n-1)}
\end{array}\right]\left[\begin{array}{l}
y_{1} \\
y_{e}
\end{array}\right]+z_{1}=\left[\begin{array}{ll}
0 & \mathbf{0}_{1 \times(n-1)}
\end{array}\right]\left[\begin{array}{l}
y_{1} \\
y_{e}
\end{array}\right]+z_{1}=z_{1}, \\
& \dot{y}_{e}=\left[\begin{array}{ll}
\mathbf{l}_{(n-1) \times 1} & S_{e(n-1) \times(n-1)}
\end{array}\right]\left[\begin{array}{cc}
0 & \mathbf{0}_{1 \times(n-1)} \\
A_{1(n-1) \times 1} & A_{e(n-1) \times(n-1)}
\end{array}\right]\left[\begin{array}{cc}
1 & \mathbf{0}_{1 \times(n-1)} \\
\mathbf{l}_{(n-1) \times 1} & S_{e(n-1) \times(n-1)}
\end{array}\right]\left[\begin{array}{l}
y_{1} \\
y_{e}
\end{array}\right] \\
& +\left[\begin{array}{ll}
\mathbf{l}_{(n-1) \times 1} & S_{e(n-1) \times(n-1)}
\end{array}\right]\left[\begin{array}{c}
1 \\
\mathbf{0}_{(n-1) \times 1}
\end{array}\right] z_{1}=\left[\begin{array}{ll}
\mathbf{l}_{(n-1) \times 1} & S_{e(n-1) \times(n-1)}
\end{array}\right]\left[\begin{array}{cc}
0 & \mathbf{0}_{1 \times(n-1)} \\
\mathbf{0}_{(n-1) \times 1} & A_{e(n-1) \times(n-1)} S_{e(n-1) \times(n-1)}
\end{array}\right]\left[\begin{array}{c}
y_{1} \\
y_{e}
\end{array}\right] \\
& +\left[\begin{array}{ll}
\mathbf{l}_{(n-1) \times 1} S_{e(n-1) \times(n-1)}
\end{array}\right]\left[\begin{array}{c}
1 \\
\mathbf{0}_{(n-1) \times 1}
\end{array}\right] z_{1}=\left[\begin{array}{ll}
\mathbf{0}_{(n-1) \times 1} & S_{e(n-1) \times(n-1)} A_{e(n-1) \times(n-1)} S_{e(n-1) \times(n-1)}
\end{array}\right]\left[\begin{array}{l}
y_{1} \\
y_{e}
\end{array}\right]+\mathbf{l}_{(n-1) \times 1} z_{1} \\
& =S_{e(n-1) \times(n-1)} A_{e(n-1) \times(n-1)} S_{e(n-1) \times(n-1)} y_{e}+\mathbf{l}_{(n-1) \times 1} z_{1}=A_{e(n-1) \times(n-1)} y_{e}+\mathbf{l}_{(n-1) \times 1} z_{1} .
\end{aligned}
$$

Furthermore, we can know

$$
\begin{aligned}
\dot{y} & =\left[\begin{array}{c}
\dot{y}_{1} \\
\dot{y}_{e}
\end{array}\right]=\left[\begin{array}{cc}
1 & \mathbf{0}_{1 \times(n-1)} \\
\mathbf{l}_{(n-1) \times 1} & S_{e(n-1) \times(n-1)}
\end{array}\right]\left[\begin{array}{cc}
0 & \mathbf{0}_{1 \times(n-1)} \\
A_{1(n-1) \times 1} & A_{e(n-1) \times(n-1)}
\end{array}\right]\left[\begin{array}{cc}
1 & \mathbf{0}_{1 \times(n-1)} \\
\mathbf{l}_{(n-1) \times 1} & S_{e(n-1) \times(n-1)}
\end{array}\right]\left[\begin{array}{l}
y_{1} \\
y_{e}
\end{array}\right] \\
& +\left[\begin{array}{cc}
1 & \mathbf{0}_{1 \times(n-1)} \\
\mathbf{l}_{(n-1) \times 1} & S_{e(n-1) \times(n-1)}
\end{array}\right]\left[\begin{array}{c}
1 \\
\mathbf{0}_{(n-1) \times 1}
\end{array}\right] z_{1}=\left[\begin{array}{cc}
0 & \mathbf{0}_{1 \times(n-1)} \\
\mathbf{0}_{(n-1) \times 1} & A_{e(n-1) \times(n-1)}
\end{array}\right]\left[\begin{array}{l}
y_{1} \\
y_{e}
\end{array}\right]+\left[\begin{array}{c}
1 \\
\mathbf{1}_{(n-1) \times 1}
\end{array}\right] z_{1}=\widetilde{A} y+\widetilde{b} z_{1},
\end{aligned}
$$




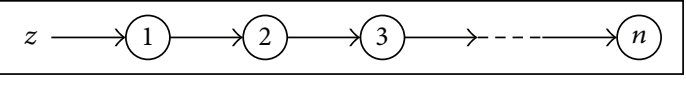

FIGURE 3: A directed path.

where

$$
\begin{aligned}
& \widetilde{A}=\left[\begin{array}{cc}
0 & \mathbf{0}_{1 \times(n-1)} \\
\mathbf{0}_{(n-1) \times 1} & A_{e(n-1) \times(n-1)}
\end{array}\right] \in R^{n \times n}, \\
& \widetilde{b}=\mathbf{1}_{n \times 1} \in R^{n \times 1} .
\end{aligned}
$$

\section{Main Results}

In this section, we first give the definition of the controllability of multiagent systems and some lemmas.

Definition 1. A nonzero state $x_{0}$ of system (4) is controllable at the initial time $t_{0}$ if there exists a finite time $t_{f}$ and a control input $z_{1}(t)$, such that $x\left(t_{0}\right)=x_{0}$ and $x\left(t_{f}\right)=0$. If any nonzero state $x_{0}$ of system (4) is controllable, then system (4) is said to be controllable.
Lemma 2 (see [2]). System (4) is controllable iff $Q_{c}$ has row full rank, where the controllability matrix of system (4) is defined as

$$
Q_{c}=\left[\begin{array}{llll}
\widetilde{b} & \widetilde{A} \vec{b} & \cdots & \widetilde{A}^{n-1} \widetilde{b}
\end{array}\right]
$$

From Lemma 2, we can have the following result.

Theorem 3. System (4) is controllable iff $\widetilde{Q}_{c}$ has row full rank, where

$$
\widetilde{Q}_{c}=\left[\begin{array}{lllll}
A_{1} & A_{e} A_{1} & \cdots & A_{e}^{(n-2)} A_{1}
\end{array}\right] .
$$

Proof. It is easy to find

$$
\widetilde{A}^{k}=\left[\begin{array}{cc}
0 & \mathbf{0}_{1 \times(n-1)} \\
\mathbf{0}_{(n-1) \times 1} & A_{e^{k}(n-1) \times(n-1)}^{k}
\end{array}\right] \in R^{n \times n},
$$

and $A_{e(n-1) \times(n-1)} \mathbf{1}_{(n-1) \times 1}=-A_{1(n-1) \times 1}$; then

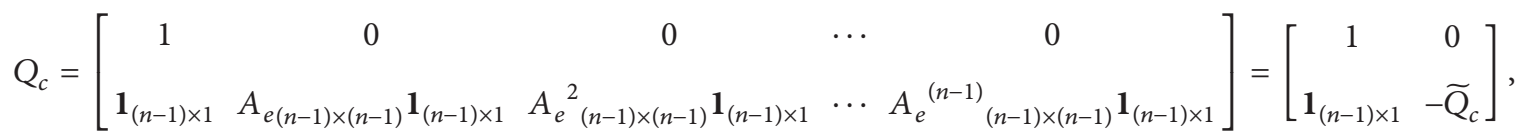

where $\widetilde{Q}_{c}=\left[\begin{array}{lllll}A_{1} & A_{e} A_{1} & \cdots & A_{e}^{(n-2)} A_{1}\end{array}\right]$. Therefore, $Q_{c}$ has full row rank if and only if $\widetilde{Q}_{c}$ has full row rank. This completes the proof.

Note that, for the multiagent system with multiple agents and high dimension, the controllable matrix of such system is too complex to calculate. But it is so easy to compute the eigenvalues of the system matrix using the $\mathrm{PBH}$ rank method by MATLAB. Next, we give a more simple and more easily checkable method.

Theorem 4 (PBH rank test). System (4) is controllable iff system (4) satisfies one of the following conditions:

(i) $\operatorname{rank}\left(s I-A_{e}, A_{1}\right)=n-1, \forall s \in \mathbb{C}$;

(ii) $\operatorname{rank}\left(\lambda_{i} I-A_{e}, A_{1}\right)=n-1$, where $\lambda_{i}, \forall i=1,2, \ldots, n-1$, is the eigenvalue of matrix $A_{e}$.

Proof. The proof is similar to that of Theorem 3 in [22], here omitted.

Since the topology graph is a directed tree, we can relabel all agents so that $A$ is a lower triangular matrix, and then $A_{1}$ is a lower triangular matrix. So we can have the following results.

Theorem 5. System (4) is controllable if coupling weights among agents are all distinct.

Proof. The proof is similar to that of Theorem 3.1 in [32], here omitted.

Corollary 6. A directed path is controllable.

Proof. From Figure 3, we have

$$
A_{e}=\left[\begin{array}{cccccc}
-a_{21} & 0 & 0 & \cdots & 0 & 0 \\
a_{32} & -a_{32} & 0 & \cdots & 0 & 0 \\
0 & a_{43} & -a_{43} & \cdots & 0 & 0 \\
\vdots & \vdots & \vdots & \ddots & \vdots & \vdots \\
0 & 0 & 0 & \cdots & -a_{n-1, n-2} & 0 \\
0 & 0 & 0 & \cdots & a_{n, n-1} & -a_{n, n-1}
\end{array}\right] .
$$



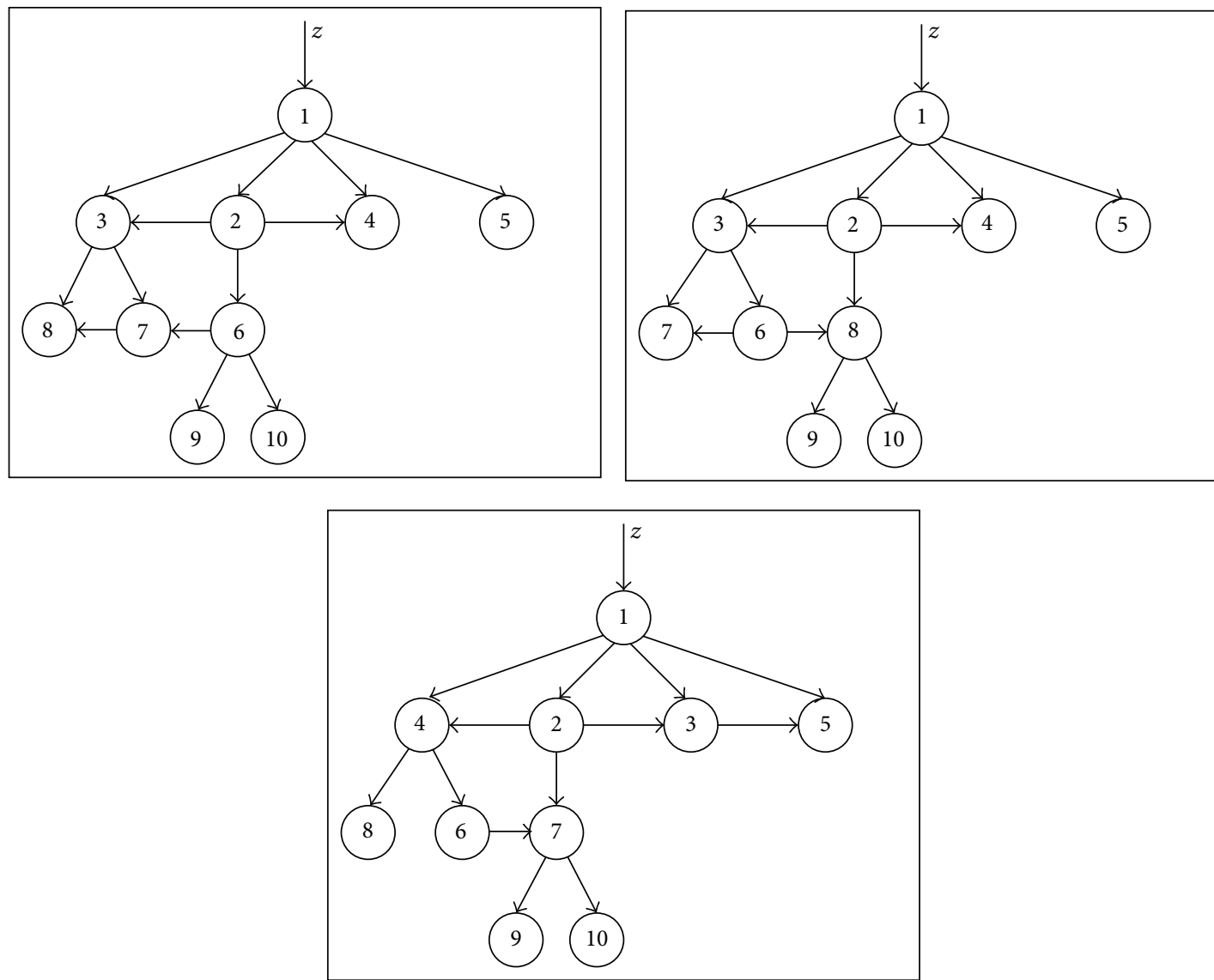

FIGURE 4: Containing a directed tree.

By computing, then

$$
\begin{aligned}
& \widetilde{Q}_{c}=\left[\begin{array}{lllll}
A_{e(n-1) \times(n-1)} \mathbf{1}_{(n-1) \times 1} & A_{e}^{2}(n-1) \times(n-1) & \mathbf{1}_{(n-1) \times 1} & \cdots & A_{e}^{(n-1)}(n-1) \times(n-1) \\
\mathbf{1}_{(n-1) \times 1}
\end{array}\right] \\
& =\left[\begin{array}{ccccc}
-a_{21} & a_{21}^{2} & \cdots & (-1)^{n-2} a_{21}^{n-2} & (-1)^{n-1} a_{21}^{n-1} \\
0 & -a_{32} a_{21} & \cdots & * & * \\
\vdots & \vdots & \ddots & \vdots & \vdots \\
0 & 0 & \cdots & -a_{n-1, n-2} \cdots a_{32} a_{21} & * \\
0 & 0 & \cdots & 0 & -a_{n, n-1} a_{n-1, n-2} \cdots a_{32} a_{21}
\end{array}\right] .
\end{aligned}
$$

Obviously, $\widetilde{Q}_{c}$ has row full rank. Hence, the directed path is controllable.

Corollary 7. A k-ary tree is uncontrollable if the weights of two children of some one agent are identical.

Furthermore, in fact, the network can be a digraph, which is not a directed tree but contains a directed tree (e.g., Figure 4). We have the following result.

Theorem 8. System (4) containing a directed tree is controllable if the following conditions are satisfied:

(i) The eigenvalues of $A_{e}$ are all distinct. (ii) The elements of $U^{-1} A_{1}$ are all nonzero, where $U=$ $\left[\varepsilon_{2}, \ldots, \varepsilon_{n}\right]$, with $\varepsilon_{i}$ being eigenvector of $A_{e}$ corresponding to eigenvalue $\lambda_{i}(i=2, \ldots, n)$.

Proof. Because $A$ is a lower triangular matrix, $A_{e}$ is also a lower triangular matrix. Then the diagonal elements of $A_{e}$ are its eigenvalue $\lambda_{i}=-\operatorname{deg}_{\text {in }}\left(v_{i}\right)(i=2,3, \ldots, n)$. Let $U \triangleq$ $\left[\varepsilon_{2}, \ldots, \varepsilon_{n}\right]$, where $\varepsilon_{i}$ is eigenvector of $A_{e}$ corresponding to eigenvalue $\lambda_{i}(i=2, \ldots, n)$. If the eigenvalues of $A_{e}$ are all distinct, then $U$ is invertible. Let $\tilde{x}=U^{-1} x$; then

$$
\dot{\tilde{x}}=U^{-1} \dot{x}=U^{-1} A_{e} U \tilde{x}+U^{-1} A_{1} u=\Lambda \tilde{x}+U^{-1} A_{1} u,
$$






Figure 5: A directed tree topology.

where $\Lambda=U^{-1} A_{e} U=\operatorname{diag}\left(\lambda_{2}, \ldots, \lambda_{n}\right)$. So

$$
\begin{aligned}
& {\left[\lambda_{i} I-\Lambda, U^{-1} A_{1}\right]} \\
& \quad=\left[\begin{array}{ccccc}
\lambda_{i}-\lambda_{2} & 0 & \cdots & 0 & b_{2} \\
0 & \lambda_{i}-\lambda_{3} & \cdots & 0 & b_{3} \\
\vdots & \vdots & \ddots & \vdots & \vdots \\
0 & 0 & \cdots & \lambda_{i}-\lambda_{n} & b_{n}
\end{array}\right] .
\end{aligned}
$$

Obviously, if conditions (i) and (ii) hold, then the system is controllable. This completes the proof.

\section{Simulation Study}

In this section, we give some numerical examples and simulations to illustrate the effectiveness of the proposed theoretical results.

4.1. Example 1. Consider a five-agent network with six agents and with a directed tree described by Figure 5. Let $a_{21}=1$, $a_{31}=2, a_{42}=3, a_{52}=4$, and $a_{63}=5$, and system (4) is defined by

$$
\begin{aligned}
A & =\left[\begin{array}{cccccc}
0 & 0 & 0 & 0 & 0 & 0 \\
1 & -1 & 0 & 0 & 0 & 0 \\
2 & 0 & -2 & 0 & 0 & 0 \\
0 & 3 & 0 & -3 & 0 & 0 \\
0 & 4 & 0 & 0 & -4 & 0 \\
0 & 0 & 5 & 0 & 0 & -5
\end{array}\right], \\
b & =\left[\begin{array}{l}
1 \\
0 \\
0 \\
0 \\
0 \\
0
\end{array}\right] .
\end{aligned}
$$

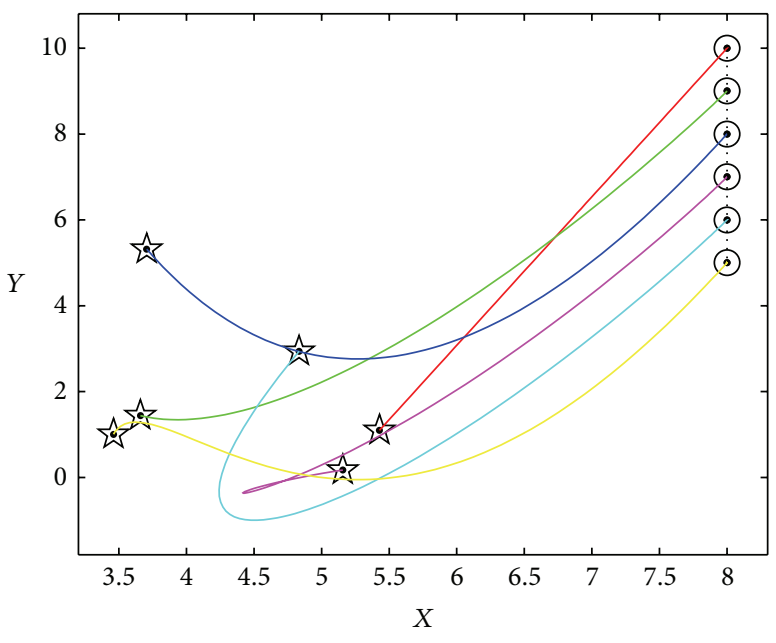

FIGURE 6: Aligning in a straight line.

Through the star transform, we can have

$$
A_{e}=\left[\begin{array}{ccccc}
-1 & 0 & 0 & 0 & 0 \\
0 & -2 & 0 & 0 & 0 \\
3 & 0 & -3 & 0 & 0 \\
4 & 0 & 0 & -4 & 0 \\
0 & 5 & 0 & 0 & -5
\end{array}\right],
$$

$$
A_{1}=\left[\begin{array}{l}
1 \\
2 \\
0 \\
0 \\
0
\end{array}\right] \text {. }
$$

By calculation, $\operatorname{Rank}\left(\widetilde{Q}_{c}\right)=5$. According to Theorem 3, the system is controllable.

Figure 6 shows the simulation result. The vertices (the agents, the black star dots) begin from random initial positions. Interconnections are depicted as lines connecting the corresponding vertices. Beginning from this initial configuration, the vertices are ultimately being controlled to a straight-line configuration.

4.2. Example 2. Consider a five-agent network containing a directed tree with six agents described by Figure 7. Let $a_{21}=$ $1, a_{31}=2, a_{32}=3, a_{41}=4, a_{42}=5, a_{51}=6$, and $a_{63}=7$, and system (4) is defined by

$$
A=\left[\begin{array}{cccccc}
0 & 0 & 0 & 0 & 0 & 0 \\
1 & -1 & 0 & 0 & 0 & 0 \\
2 & 3 & -5 & 0 & 0 & 0 \\
4 & 5 & 0 & -9 & 0 & 0 \\
6 & 0 & 0 & 0 & -6 & 0 \\
0 & 0 & 7 & 0 & 0 & -7
\end{array}\right],
$$




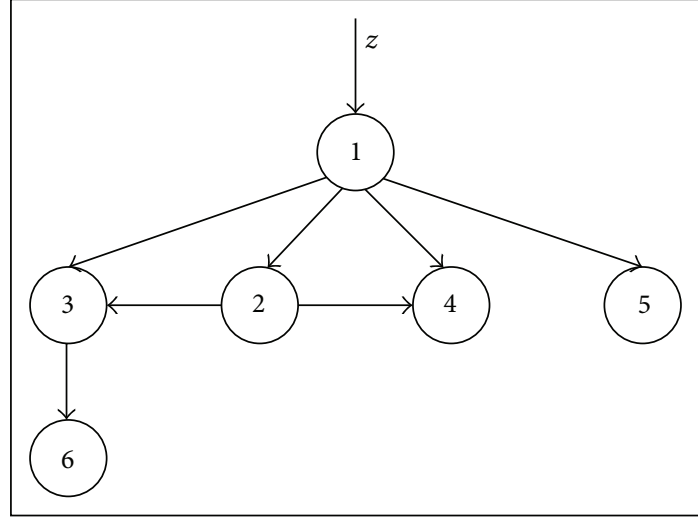

FIGURE 7: Containing a directed tree topology.

$$
b=\left[\begin{array}{l}
1 \\
0 \\
0 \\
0 \\
0 \\
0
\end{array}\right] \text {. }
$$

Through the star transform, we can have

$$
\begin{aligned}
& A_{e}=\left[\begin{array}{ccccc}
-1 & 0 & 0 & 0 & 0 \\
3 & -5 & 0 & 0 & 0 \\
5 & 0 & -9 & 0 & 0 \\
0 & 0 & 0 & -6 & 0 \\
0 & 7 & 0 & 0 & -7
\end{array}\right], \\
& A_{1}=\left[\begin{array}{l}
1 \\
2 \\
4 \\
6 \\
0
\end{array}\right] .
\end{aligned}
$$

By calculation, the eigenvalues of $A_{e}$ are $\{-7,-5,-9$, $-1,-6\}$, and

$$
U=\left[\begin{array}{ccccc}
0 & 0 & 0 & 0.6065 & 0 \\
0 & 0.2747 & 0 & 0.4549 & 0 \\
0 & 0 & 1.0000 & 0.3790 & 0 \\
0 & 0 & 0 & 0 & 1.0000 \\
1.0000 & 0.9615 & 0 & 0.5307 & 0
\end{array}\right],
$$$$
U^{-1}
$$

$$
=\left[\begin{array}{ccccc}
1.7500 & -3.5000 & 0 & 0 & 1.0000 \\
-2.7300 & 3.6401 & 0 & 0 & 0 \\
-0.6250 & 0 & 1.0000 & 0 & 0 \\
1.6489 & 0 & 0 & 0 & 0 \\
0 & 0 & 0 & 1.0000 & 0
\end{array}\right],
$$

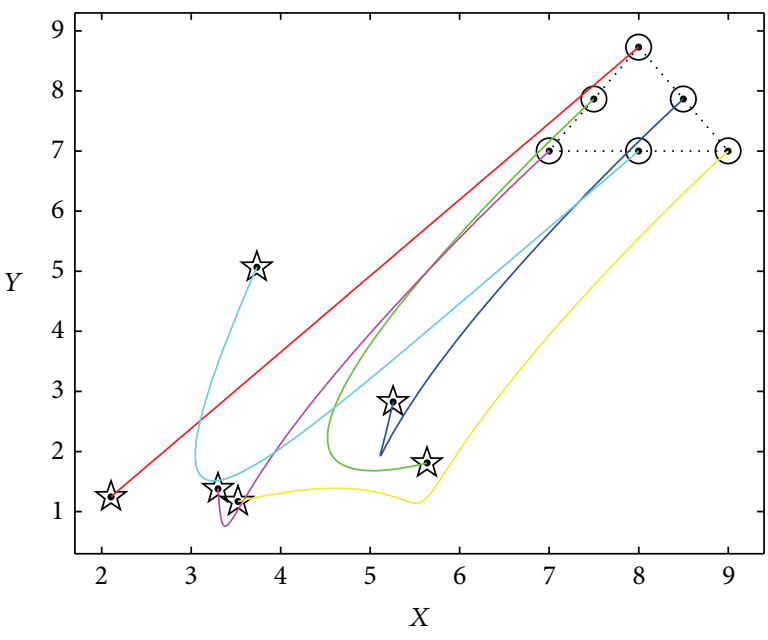

FIGURE 8: Forming a regular triangle.

and then $U^{-1} A_{1}=[5.2500,-4.5501,-3.3750,-1.6489$, $-6.0000]^{T}$. According to Theorem 8 , the system is controllable.

Figure 8 shows the simulation result, in which the agents begin from this initial configuration and are ultimately being controlled to a regular triangle configuration.

\section{Conclusion}

In this paper, we have investigated the controllability of continuous time networked systems based on consensus protocol in directed graph which is a directed tree or contains a directed tree. By applying the star transform, we can simplify the system and get an equivalent reduced-order system. We have obtained the controllability conditions of the original system from the equivalent reduced-order system.

\section{Conflict of Interests}

The authors declare that there is no conflict of interests regarding the publication of this paper.

\section{Acknowledgments}

This work was supported in part by the National Natural Science Foundation of China under Grant no. 51308005, "The-Great-Wall-Scholar" Candidate Training-Plan of North China University of Technology, Construction Plan for Innovative Research Team of North China University of Technology, and the Plan Training Project of Excellent Young Teacher of North China University of Technology, the special project of North China University of Technology (no. XN085).

\section{References}

[1] E. Wang and S. Shi, Higher Algebra, Higher Education Press, 3rd edition, 2003. 
[2] D. Zheng, Linear System Theory, Tsinghua University Press, Beijing, China, 2nd edition, 2002.

[3] M. Cao, S. Zhang, and M. K. Camlibel, "A class of uncontrollable diffusively coupled multiagent systems with multichain topologies," IEEE Transactions on Automatic Control, vol. 58, no. 2, pp. 465-469, 2013.

[4] X. Wang, X. Li, and G. Chen, Network Science: An Introduction, Higher Education Press, 2012.

[5] B. Liu, X. Wang, H. Su, H. Zhou, Y. Shi, and R. Li, "Adaptive synchronization of complex dynamical networks with timevarying delays," Circuits, Systems, and Signal Processing, vol. 33, no. 4, pp. 1173-1188, 2014.

[6] A.-H. Zhang, S.-J. Zhang, J. Chen, H.-B. Guo, X.-R. Kong, and F. Wang, "Graph-theoretic characterization of controllability for multi-agent systems," Control and Decision, vol. 26, no. 11, pp. 1621-1631, 2011.

[7] R. Olfati-Saber and R. M. Murray, "Consensus problems in networks of agents with switching topology and time-delays," IEEE Transactions on Automatic Control, vol. 49, no. 9, pp. 15201533, 2004.

[8] H. Su, Z. Rong, M. Z. Q. Chen, X. Wang, G. Chen, and H. Wang, "Decentralized adaptive pinning control for cluster synchronization of complex dynamical networks," IEEE Transactions on Cybernetics, vol. 43, no. 1, pp. 394-399, 2013.

[9] Z. Wang and J. Zhi, "Controllability of discrete-time multi-agent systems with time-delay in state," Journal of Qingdao University, vol. 24, no. 2, pp. 1-5, 2009.

[10] Z. Ji, H. Lin, and T. H. Lee, "A graph theory based characterization of controllability for multi-agent systems with fixed topology," in Proceedings of the 47th IEEE Conference on Decision and Control, pp. 5262-5267, Cancun, Mexico, December 2008.

[11] H. Su, G. Jia, and M. Z. Chen, "Semi-global containment control of multi-agent systems with input saturation," IET Control Theory \& Applications, vol. 8, no. 18, pp. 2229-2237, 2014.

[12] M. Ji and M. Egerstedt, "A graph-theoretic characterization of controllability for multi-agent systems," in Proceedings of the American Control Conference (ACC '07), pp. 4588-4593, IEEE, New York, NY, USA, July 2007.

[13] Y. Zheng, Y. Zhu, and L. Wang, "Consensus of heterogeneous multi-agent systems," IET Control Theory \& Applications, vol. 5, no. 16, pp. 1881-1888, 2011.

[14] S. Jafari, A. Ajorlou, A. G. Aghdam, and S. Tafazoli, "On the structural controllability of multi-agent systems subject to failure: a graph-theoretic approach," in Proceedings of the 49th IEEE Conference on Decision and Control (CDC '10), pp. 4565-4570, December 2010.

[15] H. Su, N. Zhang, M. Z. Chen, H. Wang, and X. Wang, "Adaptive flocking with a virtual leader of multiple agents governed by locally Lipschitz nonlinearity," Nonlinear Analysis. Real World Applications, vol. 14, no. 1, pp. 798-806, 2013.

[16] Y.-Y. Liu, J.-J. Slotine, and A.-L. Barabási, "Controllability of complex networks," Nature, vol. 473, no. 7346, pp. 167-173, 2011.

[17] H. G. Tanner, "On the controllability of nearest neighbor interconnections," in Proceedings of the IEEE Conference on Decision and Control, vol. 3, pp. 2467-2472, 2004.

[18] B. Liu, T. Chu, L. Wang, and G. Xie, "Controllability of a leaderfollower dynamic network with switching topology," IEEE Transactions on Automatic Control, vol. 53, no. 4, pp. 1009-1013, 2008.
[19] B. Liu, H. Feng, L. Wang et al., "Controllability of second-order multiagent systems with multiple leaders and general dynamics," Mathematical Problems in Engineering, vol. 2013, Article ID 587569, 6 pages, 2013.

[20] F. Jiang, L. Wang, G. Xie, Z. Ji, and Y. Jia, "On the controllability of multiple dynamic agents with fixed topology," in Proceedings of the American Control Conference (ACC '09), pp. 5665-5670, June 2009.

[21] B. Liu, T. Chu, L. Wang, and G. Xie, "Controllability of a class of multi-agent systems with a leader," in Proceedings of the American Control Conference (ACC '06), pp. 2844-2849, IEEE, Minneapolis, Minn, USA, June 2006.

[22] B. Liu, H. Su, R. Li, D. Sun, and W. Hu, "Switching controllability of discrete-time multi-agent systems with multiple leaders and time-delays," Applied Mathematics and Computation, vol. 228, pp. 571-588, 2014.

[23] B. Liu, W. Hu, J. Zhang, and H. Su, "Controllability of discretetime multi-agent systems with multiple leaders on fixed networks," Communications in Theoretical Physics, vol. 58, no. 6, pp. 856-862, 2012.

[24] K. Liu, G. Xie, W. Ren, and L. Wang, "Consensus for multiagent systems with inherent nonlinear dynamics under directed topologies," Systems \& Control Letters, vol. 62, no. 2, pp. 152-162, 2013.

[25] H. Su, M. Z. Q. Chen, J. Lam, and Z. Lin, "Semi-global leaderfollowing consensus of linear multi-agent systems with input saturation via low gain feedback," IEEE Transactions on Circuits and Systems-I. Regular Papers, vol. 60, no. 7, pp. 1881-1889, 2013.

[26] Y. Sun and L. Wang, "Consensus of multi-agent systems in directed networks with nonuniform time-varying delays," IEEE Transactions on Automatic Control, vol. 54, no. 7, pp. 1607-1613, 2009.

[27] J. Yu and L. Wang, "Group consensus of multi-agent systems with directed information exchange," International Journal of Systems Science, vol. 43, no. 2, pp. 334-348, 2012.

[28] H. Su, M. Z. Q. Chen, X. Wang, and J. Lam, "Semiglobal observer-based leader-following consensus with input saturation," IEEE Transactions on Industrial Electronics, vol. 61, no. 6, pp. 2842-2850, 2014.

[29] J. Yu and L. Wang, "Group consensus in multi-agent systems with switching topologies and communication delays," Systems \& Control Letters, vol. 59, no. 6, pp. 340-348, 2010.

[30] Y. Zheng and L. Wang, "Containment control of heterogeneous multi-agent systems," International Journal of Control, vol. 87, no. 1, pp. 1-8, 2014.

[31] H. Su and M. Z. Q. Chen, "Multi-agent containment control with input saturation on switching topologies," IET Control Theory \& Applications, vol. 9, no. 3, pp. 399-409, 2015.

[32] D. Guo, G. Yan, and Z. Lin, "Distributed verification of controllability for weighted out-tree based topology," in Proceedings of the 2012 American Control Conference, pp. 1507-1512, June 2012.

[33] L. Hsu and C. Lin, Graph Theory and Interconnection Networks, CRC Press, 2008. 


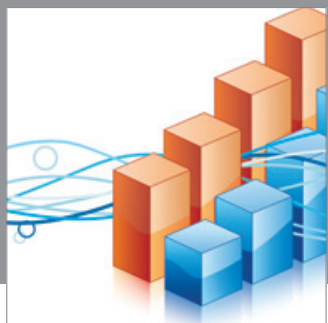

Advances in

Operations Research

mansans

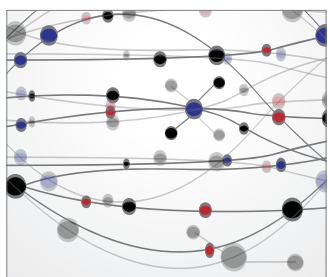

The Scientific World Journal
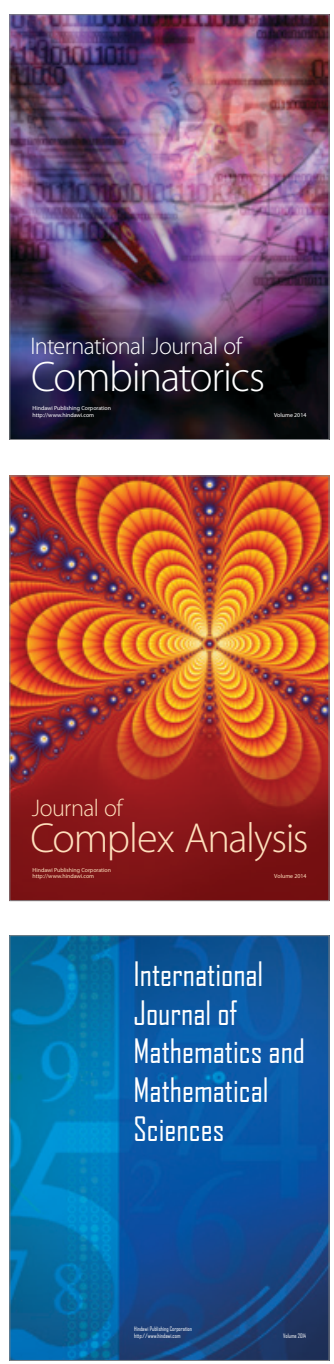
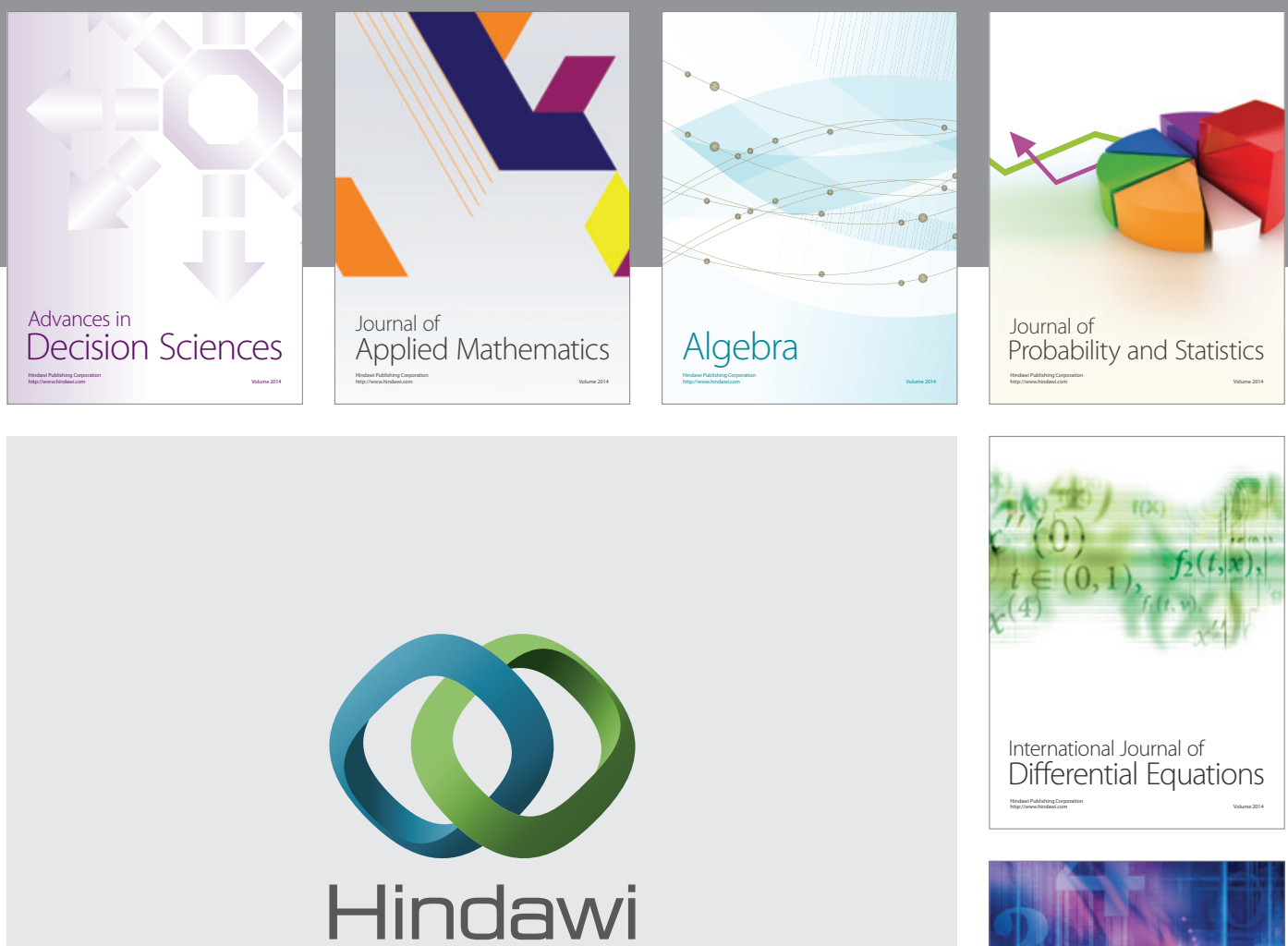

Submit your manuscripts at http://www.hindawi.com
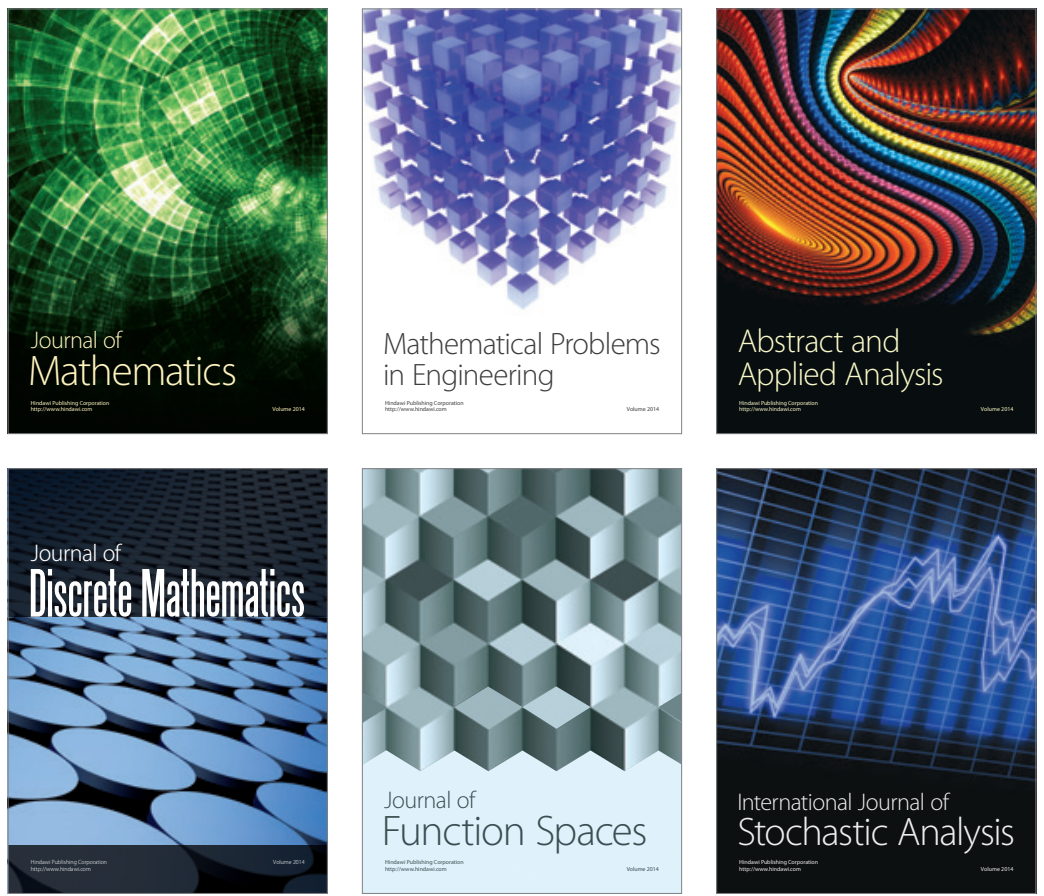

Journal of

Function Spaces



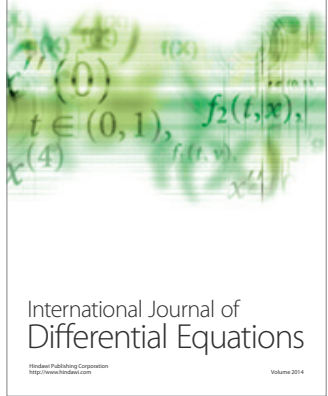
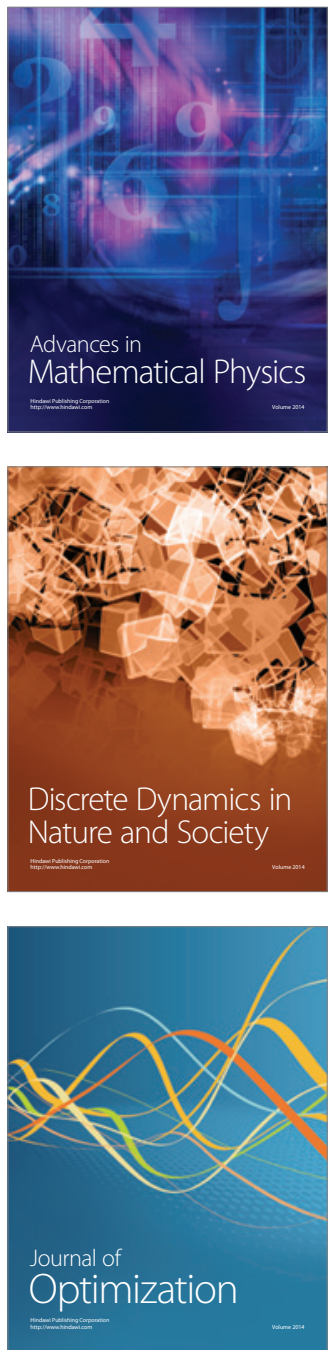